Relato de Caso

\title{
Estratégia de aproveitamento de dentes envolvidos por cistos em
} pacientes pediátricos

\author{
Strategy of utilization of teeth involved by cysts in pediatric patients
}

Thalles Moreira Suassuna1; Isadora Serrão Wanderley²; Yuri Barbosa Alves²; Ávilla Pessoa Aguiar3; Fábio Correia Sampaio4; José Wilson Noleto4.

${ }^{1}$ Mestrando em Odontologia da Universidade Federal da Paraíba, João Pessoa - Paraíba - Brasil ${ }^{2}$ Acadêmico de Odontologia da Universidade Federal da Paraíba, João Pessoa - Paraíba - Brasil ${ }^{3}$ Acadêmica de Odontologia no Instituto de Educação Superior da Paraíba, João Pessoa Paraíba - Brasil

${ }^{4}$ Professor do curso de Odontologia da Universidade Federal da Paraíba, João Pessoa - Paraíba - Brasil

Autor e endereço para correspondência:

Thalles Moreira Suassuna, Rua Eutiquiano Barreto, $n^{\circ} 815$, CEP: 58038-311 Email: thallesms_@hotmail.com.

\section{Resumo}

Introdução: Cisto é uma cavidade patológica preenchida por líquido ou material semisólido e revestida por epitélio. Cistos ósseos revestidos por epitélio são quase exclusividade dos ossos gnáticos e são relativamente comuns na rotina odontológica. Esta patologia tende a aumentar lentamente de tamanho, possivelmente em resposta ao aumento da pressão osmótica luminal. Os Cistos Odontogênicos são mais encontrados em pacientes entre 10 e 30 anos, mas podem acometer indivíduos na primeira década de vida e envolver múltiplos elementos dentários ainda em formação. Relato de caso: Dois pacientes pediátricos com história de trauma e necrose pulpar não diagnosticada a tempo, em incisivo central decíduo. Apresentaram retardo na erupção dos elementos permanentes adjacentes e retenção prolongada do incisivo necrosado. Radiograficamente era vista herniação dos elementos permanentes em formação. Ambos os casos foram tratados através de biopsia incisional, descompressão por 6 meses, enucleação conservadora e tratamento ortodôntico. Conclusão: Os cistos inflamatórios podem ser removidos de forma menos agressiva, mantendo intactos os elementos dentários envolvidos, desde que estes apresentem viabilidade.

Descritores: Cistos Ósseos, Cistos Odontogênicos, Germe de dente. 


\begin{abstract}
Introduction: Cyst is a pathological cavity filled with liquid or semi-solid material and coated with epithelium. Epithelium-lined bone cysts are almost exclusive to gnathic bones and are relatively common in routine dentistry. This pathology tends to increase flexibility, the possibility of increasing luminal osmotic pressure. Odontogenic Cysts are More Distant Between 10 and 30 Years, can be included in the first decade of life and present the results of the processes still in formation. Case report: Two pediatric patients with a history of trauma and pulpal necrosis undiagnosed in time, in deciduous central incisor. They presented delayed eruption of the adjacent permanent elements and prolonged retention of the necrotic incisor. Radiographically it was seen herniation of the permanent elements in formation. Both cases were treated through incisional biopsy, decompression for 6 months, conservative enucleation and orthodontic treatment. Conclusion: The inflammatory cysts can be removed in a less aggressive way, keeping intact the dental elements involved, as long as they present viability.
\end{abstract}

Keywords: BoneCysts, OdontogenicCysts, Tooth Germ..

\title{
Introdução
}

Cisto é uma cavidade patológica preenchida por líquido ou material semi-sólido e revestida por epitélio. Cistos ósseos verdadeiros são quase exclusividade dos ossos gnáticos e são relativamente comuns na rotina odontológica ${ }^{1,2,3}$.

Estas lesões podem ser de Desenvolvimento, quando supostamente se originam da inclusão de epitélio ao longo das linhas de fusão dos processos embrionários. Ou podem ser Odontogênicos (CO) quando o seu epitélio é derivado do epitélio odontogênico. Nesta última categoria eles ainda podem ser classificados como Inflamatórios ou de Desenvolvimento (Quadro $1)^{2}$.

Os remanescentes epiteliais presentes na maxila e mandíbula são originados do ectoderma que reveste os processos embrionários que irão formar a face ou de tecido epitelial que participa na odontogênese ${ }^{10}$. A simples presença desses restos epiteliais seria insuficiente para explicar a formação de um cisto. É necessária a ação de um agente inflamatório, por exemplo, capaz de estimular e determinar a proliferação desses remanescentes ${ }^{10}$. Tal condição é 
frequente nos maxilares, onde infecções e traumas são capazes de desencadear a resposta inflamatória ${ }^{3,4,10,11}$.

Os COs tendem a aumentar lentamente de tamanho, possivelmente em resposta ao aumento da pressão osmótica luminal. Genericamente estas lesões podem: ser assintomáticas, variar de milímetros a alguns centímetros e costumam causar deslocamento de estruturas adjacentes (como dentes, raízes e nervos) ${ }^{4}$. Radiograficamente costuma ser visível uma imagem radiolúcida, unilocular, de limites bem definidos e rodeada por uma margem de osso esclerótico ${ }^{4,5,6}$.

Quando nos maxilares, os cistos são mais encontrados em pacientes entre 10 e 30 anos, mas podem acometer indivíduos na primeira década de vida e assim envolver múltiplos germes dentários. As entidades mais relacionadas a esse fenômeno são o Cisto Dentígero e, principalmente, o Cisto Periapical ${ }^{7,8,9}$.

Nos casos que serão apresentados, foi identificada a fonte de inflamação que originou os cistos. Ambos os pacientes tinham história de trauma dental na primeira infância e sinais de necrose pulpar em um incisivo decíduo, semelhante a caso relatado ${ }^{12}$.

O objetivo deste trabalho é, através de casos clínicos, propor uma abordagem de tratamento que vise o aproveitamento dos dentes envolvidos..

\section{Relato dos Casos}

Caso I: Paciente NRFS, 10 anos, gênero feminino e com história de trauma dentário há 4 anos. Clinicamente evidenciava abaulamento vestibular e palatino, atraso na esfoliação dos elementos 61, 62 e 63 e escurecimento da coroa do 61. Radiograficamente observamos herniação do 21, 22 e 23 em direção a órbita e grande imagem radiolúcida circunscrita medindo cerca de $4 \mathrm{~cm}$ (Figura 1).

A conduta adotada a princípio foi a biopsia incisional e descompressão. O diagnóstico apontado foi de Cisto Periapical. Após 6 meses e diante da regressão parcial do cisto e melhora no posicionamento dos elementos, foi realizada enucleação, remoção do 23 (que estava mal posicionado) e mantendo os demais elementos, sem causar abalo ou descolamento do seu capuz.

A partir do terceiro mês de pós-operatório foi encaminhada a ortodontia para manutenção de espaço e monitoramento da erupção, para intervir quando necessário. Atualmente a paciente encontra-se em fase de erupção dos elementos. 

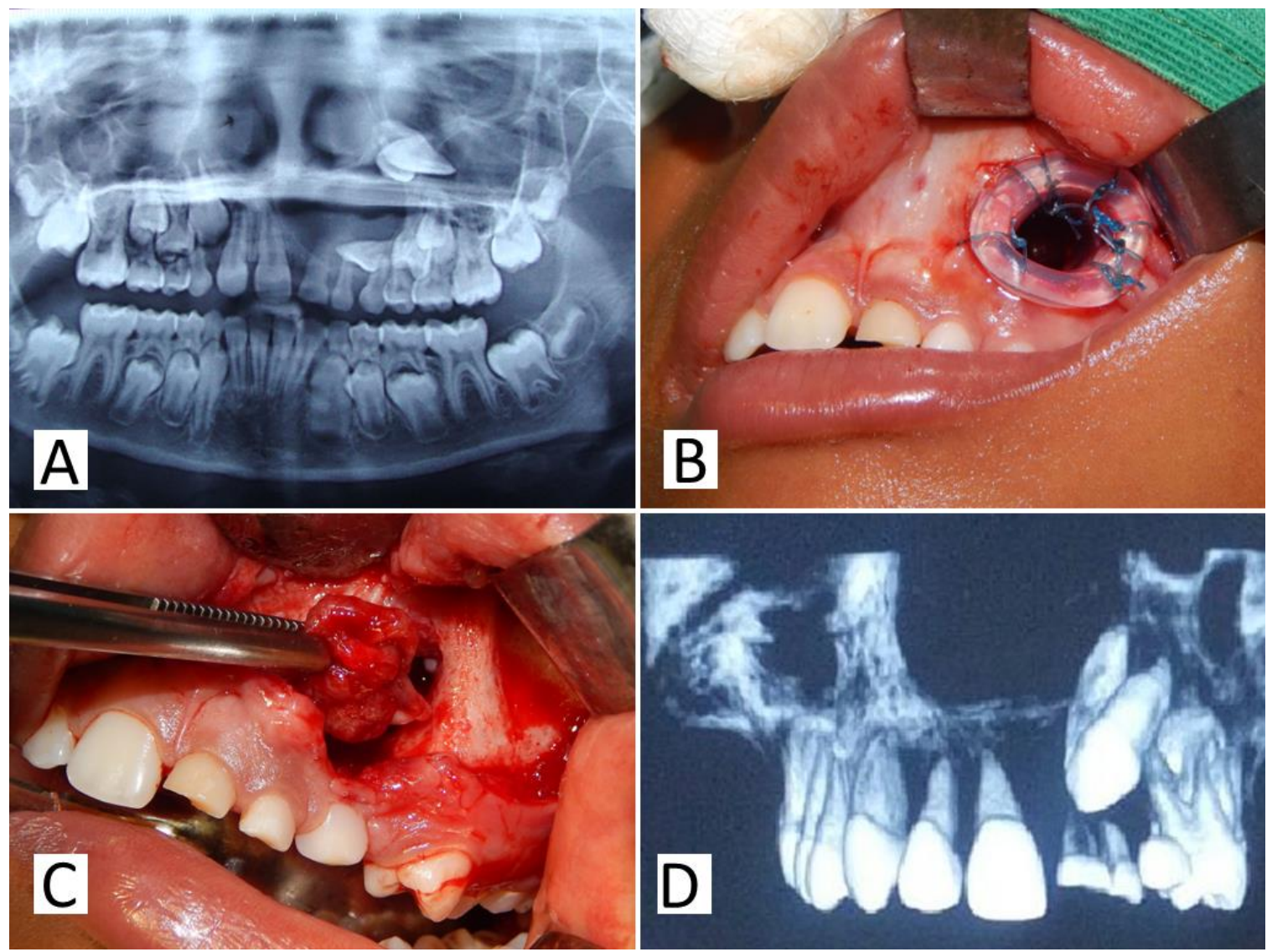

Figura 1: A) Raio x Panorâmico antes do tratamento evidência extensa imagem radiolúcida, de limites bem definidos rechaçando os elementos 21 e 22 em direção à órbita. B) Instalação de dreno no mesmo tempo cirúrgico da biópsia incisional. C) Enucleação da lesão 6 meses após a descompressão. Observe que o epitélio do cisto está espesso e o defeito ósseo é bem limitado.

D) Reconstrução tridimensional da região um ano após a remoção da lesão. Nota-se que os elementos dentários inclusos se encontram em posicionamento favorável à erupção.

Caso II: Paciente PHLA, sete anos, gênero masculino e também com história de trauma local prévio e necrose pulpar. Apresentava atraso na erupção do11 e abaulamento vestibular sob a mucosa. O tratamento instituído foi idêntico ao primeiro caso, no entanto todos os elementos apresentaram posicionamento favorável e estão sendo aproveitados. Este paciente também encontra-se em fase de finalização ortodôntica. 

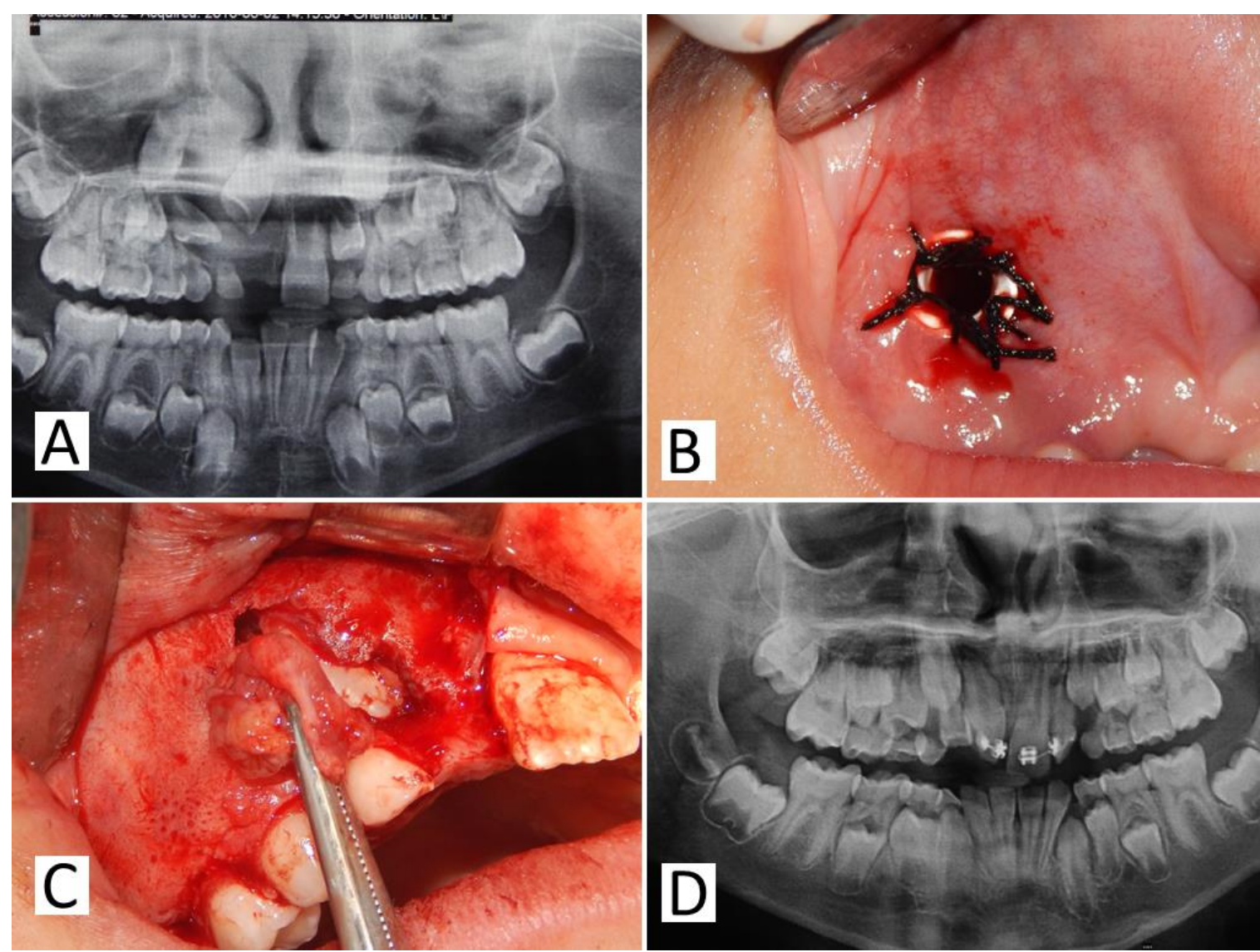

Figura 2: A) Raio x panorâmico mostra imagem radiolúcida envolvendo a coroa do 11, que está com seu processo de erupção atrasado. B) Dispositivo de descompressão, improvisado a partir de uma taça de borracha. C) Enucleação da lesão, mantendo os elementos dentários permanentes. D) Raio $x$ panorâmico 16 meses após a enucleação evidencia que os elementos herniados pelo cisto agora se encontram praticamente dentro do alvéolo.

\section{Discussão}

As formas de tratamento indicadas para os cistos são, basicamente, a enucleação (remoção total da lesão cística) e a descompressão (qualquer técnica que alivie a pressão dentro do cisto $)^{1,7,8}$. Embora estas modalidades de tratamento possam ser utilizadas isoladamente, 0 tratamento mais adequado para grandes cistos é a associação de ambos os métodos, principalmente quando se deseja minimizar danos a estruturas importantes ${ }^{8,13}$.

A descompressão é feita através de uma pequena abertura na parede do cisto, que se mantém aberta por meio de um dreno ${ }^{13,14}$. Esta técnica cessa o crescimento do cisto e, em muitas 
vezes, promove a sua regressão e um crescimento ósseo centrípeto ${ }^{14}$. Além disso, permite que elementos dentários envolvidos possam adquirir posicionamento mais favorável para a futura erupção. Dessa maneira, a cirurgia de enucleação torna-se mais segura e conservadora para o paciente pa, $^{13,14}$.

Quando se deseja preservar os dentes, é imperativo que durante o procedimento cirúrgico se remova todo o epitélio do cisto sem, no entanto, remover os capuzes pericoronários e sem deslocar os elementos, para não causar traumas adicionais nem comprometimento ao aporte vascular ${ }^{15}$. Esta abordagem mais conservadora só é possível porque os cistos inflamatórios e o dentígero tem natureza benigna e baixas taxas de recidiva ${ }^{7,8,15}$. Neste contexto é imperativo saber qual a variante do cisto odontogênico está sendo tratada, pois algumas entidades, como o Ceratocisto Odontogênico, possuem taxas de recorrência importantes ${ }^{3,14}$.

Vários autores concordam que o planejamento do tratamento deve incluir uma avaliação da viabilidade dos dentes. Fatores como posicionamento do elemento e a presença de deformidades no elemento podem deixar o prognóstico duvidoso e, em alguns casos, a exodontia poderá ser indicada no mesmo ato da enucleação $0^{6,14,15}$.

Os dentes em formação possuem força de erupção e se eles estiverem com um eixo favorável é esperado uma boa movimentação em direção ao alvéolo. Isto acontece especialmente na maxila, que é um osso esponjoso e o movimento dos dentes é a favor da gravidade ${ }^{14,15}$.

A ortodontia tem papel importante nestes casos para avaliar se o dente ainda é viável. Além disso, logo após a enucleação é importante iniciar o acompanhamento ortodôntico para manter o alinhamento no arco e o correto espaço pros dentes. Deve-se monitorar os elementos para observar o estágio de formação radicular e se a sua posição está progredindo. Em casos onde haja fechamento do ápice ou paralisação do movimento, virá a ser necessária a abordagem do elemento para colocação de dispositivo para tracionamento ${ }^{10,14,15}$.

\section{Conclusão}

Tais resultados nos permitem concluir que, pelo fato de a maioria dos cistos terem baixas taxas de recidiva, podem ser removidos de forma conservadora, mantendo-se os elementos dentários envolvidos, desde que estes apresentem viabilidade. Além disso, fica evidente a necessidade de acompanhamento multidisciplinar para maximização do resultado. 


\section{Referências}

1. Nagori SA, Jose A, Bhutia O, Roychoudhury A. Large pediatric maxillary dentigerous cysts presenting with sinonasal and orbital symptoms: A case series. Ear Nose Throat J. 2017;96(45):29-34.

2. Martin LHC, Speight PM.Odontogenic cysts: anup date. Diagnostic Histopathology. 2017;23(6):260-5.

3. Arce K, Streff CS, Ettinger KS. Pediatric Odontogenic Cysts oft He Jaws. Oral Maxillofacial Surg Clin N Am. 2016;28(1):21-30.

4. Sharma S, Sharma V, Passi D, Srivastava D, Grover S, Dutta SR. Large Periapical or Cystic Lesions in Association with Roots Having Open Apices Managed Nonsurgically Using 1-step Apexification Based on Platelet-rich Fibrin Matrix and Biodentine Apical Barrier: A Case Series. J Endod. 2018;44(1):179-185.

5. Abdelwahab M, Elfattah AMA, Khafagy YW, Degwi AE. Endoscopic enucleation of large jaw cysts: Promising outcomes. Auris Nasus Larynx. 2018; 45(3):578-584.

6. Pitcher B, Alaqla A, Noujeim M, Wealleans JA, Kotsakis G, Chrepa V. Binary Decision Trees for Pre operative Periapical Cyst Screening Using Cone-beam Computed Tomography. J Endod. 2017;43(3):383-388.

7. Li N, Gao X, Xu Z, Chen Z, Zhu L, Wang J, Liu W. Prevalence of develop mental odontogenic cysts in children and adolescents withem phasison dentigerous cyst. Acta Odontologica Scandinavica. 2014; 72(8):795-800.

8. $\mathrm{Hu} \mathrm{YH}$, Chang $\mathrm{YL}$, Tsai $\mathrm{A}$. Conservative treatment of dentigerous cyst associated with primary teeth. Oral Surg Oral Med Oral Pathol Oral. 2011;112(6):5-7.

9. Dhupar A, Yadav S, Dhupar V, Mittal HC, Malik S, Rana P. Bi-maxillary dentigerous cyst in a non-syndromic child - review of literature with a case presentation. J Stomatol Oral Maxillofac Surg. 2017;118(1):45-8.

10. Ribeiro ET, Bridi MP, Pereira TCR, Velloso TRG, Salim MAA, Bertollo RM, Maia RMLC, Barros LAP, Silva DN. Impacted teeth associated with odontogenic cysts and tumors. Rev. Bras. Pesq. Saúde. 2015;17(2):78-88.

11. Gondim JO, Giro EMA, Moreira Neto JJS, Coldbella CR, Bolini PDA, Gaspar AMM. Sequela es in permanent teeth following traumatic injuries to primary predecessors and clinical implication. Rev Gaúcha Odontol. 2011;59(9):113-120. 
12. Jopson J, Somasundaran S, Courtney D. Unusual presentation of an odontogenic cyst in a child. Journal of Paediatrics and Child Health. 2017;53:923-927.

13. Rodrigues JT, Antunes HS, Armada L, Pires FR. Influence of surgical decompression on the expression of inflammatory and tissue repair biomarkers in periapical cysts. Oral Surg Oral Med Oral Pathol Oral Radiol. 2017;124(6):561-567.

14. Alpy A, Tournaire L, Vaysse F, Marchal-Sixou C, Lhomme A, Courtois B. Interest of decompression in orthodontics: Case reportof a keratocyst during child hood. International Orthodontics. 2017;15(2):238-250.

15. Maltoni I, Santucci G, Maltoni M, Zoli L, Perri A, Gracco A. Recovering teeth from a large dentigerous cyst: A case report. International Orthodontics. 2015;13(2): 232-244. 